\title{
Contribuição da intervenção nutricional no tratamento da hipertensão arterial: experiência de uma equipe interdisciplinar
}

The contribution of nutritional intervention in the treatment of arterial hypertension: an experience of an interdisciplinary team

Odaleia Barbosa de Aguiar ${ }^{1}$

Vera Lucia Rabello de Castro Halfoun ${ }^{2}$

Renata Couto Falcão Gomes ${ }^{3}$

\section{RESUMO}

Avaliamos a contribuição da dieta sobre peso, pressão arterial (PA), aderência ao tratamento e qualidade de vida (QV) nos pacientes dos programas de hipertensão e de diabetes acompanhados por equipe interdisciplinar em unidade primária de saúde. Estudamos 116 pacientes, com idades entre 20 e 65 anos, por seis meses e aplicamos questionários padronizados para avaliar QV e aderência ao tratamento. Foram analisados sexo, diagnóstico, atividade física, faixa etária, índice de massa corporal, consumo alimentar e variação (DP) entre peso inicial $\left(P_{j}\right)$ e final $\left(P_{f}\right)$ de toda a amostra, dos obesos, daqueles que aderiram ou não ao tratamento e dos que controlaram ou não a PA. Utilizamos teste Mann Whitney para comparações entre subgrupos e, para dados seqüenciais, teste de Dunn ( $p<0,05$ exigido). Foi feita análise multivariada, comparando escores de QV com idade, $P_{f}$, peso relativo $\left[\left(P_{f} / P_{i}\right) \times 100\right]$ e DP. Foi estudado um grupo de 65 mulheres e 51 homens, predominantemente entre 45 e 64 anos, com hipertensão (33,7\%), diabetes mellitus (8,6\%) ou ambos (57,7\%). Dentre os analisados, $81 \%$ eram sedentários; $52,5 \%$ consumiam frutas e $45,6 \%$, vegetais, diariamente. O índice de massa corporal encontrado foi $29,34 \pm 5,22 \mathrm{~kg} / \mathrm{m}^{2}$, maior entre os de 45 a 54 anos $\left(30,71 \pm 5,5 \mathrm{~kg} / \mathrm{m}^{2}\right)$. Houve redução significativa do peso $(p<0,0001)$ nos obesos $(42,24 \%)$ e nos que controlaram a pressão. Entre os que aderiram à dieta e aos exercícios físicos ocorreu maior queda de peso. Não houve associação importante entre escores de QV e resposta ponderal. Consideramos que a abordagem dietética em um modelo de serviço interdisciplinar contribuiu de modo eficaz para a melhora dos pacientes hipertensos e diabéticos tratados em unidade primária de saúde.

PALAVRAS-CHAVE:

- Hipertensão;

- Terapia Nutricional;

- Relações Interprofissionais;

- Qualidade de Vida;

\section{KEY-WORDS:}

- Hypertension;

- Nutrition Therapy;

- Interprofessionals Relations;

- Quality of Life;

\footnotetext{
${ }_{1}^{1}$ Nutricionista UFRJ, mestre em Nutrição Humana, UFRJ, Professora-assistente, UERJ

Médica, doutora em Medicina, Professora-Adjunta, UFRJ.

3 Mestranda em Nutrição Humana, UNB, Nutricionista, UFRJ.
} 


\section{ABSTRACT}

Diet effects on weight, blood pressure (BP), treatment adherence and quality of life (QL) were studied in patients with arterial hypertension and diabetes mellitus followed by interdisciplinary team in one primary health care unit. 116 patients (2065 years old) were selected, at least 7 visits demanded. Treatment adherence and QL were analyzed by standard questionnaires. Sex, age, body mass index (BMI), physical activity, fruits and vegetables consume were registered. Final weight (Pf), initial and final weight variation $(D P)$ and relative weight $\left[\left(P_{P} / P_{j}\right) \times 100\right]$ were studied in all patients as well as among the obese ones and those who had adhered to treatment and controlled $B P$. Comparisons among groups and sequential data were done by Mann Whitney and Dunn tests, respectively ( $p<0,05$ demanded). Multiple regression was used comparing $Q L$ scores with $P_{f}, P_{r}$ and DP. 65 women and 51 men were studied (most 45-64 years old): $33.7 \%$ had arterial hypertension, $8.6 \%$ diabetes mellitus, and $57.7 \%$ both; $81 \%$ had not used to make regular physical exercises and $52.5 \%$ to consume fruits and $45.6 \%$ vegetables daily. Mean BMI was $29.34 \pm 5.22 \mathrm{~kg} /$ $m^{2}$. There was significant weight reduction, mainly among the obese ones ( $p<0,0001)$, and among those who brought their BP to normal values. Higher weight reduction was obtained in those who adhered to diet and/or physical exercises than others that did not do them. There was no important associations between weight parameters and QL scores. Diet approach, in an interdisciplinary health care model, was efficient to improve response to treatment.

\section{I - Introdução}

Nos últimos anos, o ato de se alimentar vem ganhando destaque na sociedade, um novo cenário está sendo construído para os alimentos e a ênfase na alimentação saudável passa a fazer parte das orientações dos profissionais de saúde. Conversas sobre dietas, excesso de peso, valor nutricional dos alimentos, produtos diet/light e o debate que ora se instala sobre os transgênicos fazem parte das principais temáticas assumidas pelas sociedades em geral e, em especial, pela área da saúde.

No campo da saúde, os hábitos relacionados ao estilo de vida, como dieta rica em calorias, gorduras saturadas, colesterol e sódio, têm sido apontados entre os fatores de risco considerados de maior importância na etiologia da doença cardiovascular. A dieta, por sua vez, está relacionada à patogênese da chamada síndrome metabólica, incluindo as dislipidemias, a obesidade e o diabetes. ${ }^{1,2}$

A intervenção nutricional nos programas de controle de hipertensão arterial sistêmica (HAS) e diabetes mellitus tipo 2 (DM2) tem como objetivo orientar o paciente a alcançar seu peso desejável por meio da seleção dos alimentos que compõem sua dieta; manter normais os níveis sangüíneos de glicose e contribuir para a melhoria de seu estado de saúde geral, prevenindo ou retardando as complicações relacionadas à nutrição.

Um estudo preliminar desenvolvido pelo Programa de Atenção Primária à Saúde (PAPS) da Faculdade de Medicina da UFRJ, em 1998, mostrou altos índices de abandono ao Programa de Hipertensão (60\%) e baixo percentual de pacientes diabéticos $(2,8 \%)$ atendidos em um Centro Municipal de Saúde localizado no município 
do Rio de Janeiro (CMS).

A partir de 1999, a Faculdade de Medicina da UFRJ iniciou um projeto de atendimento à população de pacientes hipertensos e de diabéticos atendidos em um $\mathrm{CMS}^{3}$. O modelo proposto tem como princípio o atendimento interdisciplinar no acompanhamento de hipertensos e/ou diabéticos, uma vez que esse tipo de assistência tem menor custo que 0 atendimento tradicional. Dessa forma, pretende-se garantir o controle da(s) doença(s), aumentar a aderência ao tratamento e reduzir o abandono às consultas, por meio da maior satisfação dos usuários e da melhora da sua qualidade de vida.

O objetivo deste trabalho foi avaliar a resposta ponderal dos pacientes inseridos neste modelo de atendimento, relacionando-a com a resposta pressórica daqueles hipertensos, com a aderência ao tratamento realizado e com a sua qualidade de vida.

Este projeto foi submetido ao Comitê de Ética em Pesquisa no Hospital Universitário Clementino Fraga Filho do Centro de Ciências da Saúde da UFRJ, tendo parecer favorável no dia 06/05/1999 e sendo registrado no protocolo de pesquisa número 36/99.

\section{II - Metodologia}

Constituíram nossa casuística os pacientes que preenchiam os critérios de inclusão nos programas de hipertensão arterial e de diabetes mellitus de acordo com as normas da Secretaria Municipal de Saúde do Rio de Janeiro (SMS-RJ), os quais foram acompanhados de maio de 1999, no Projeto Assistencial Interdisciplinar de atendimento aos pacientes com HAS e DM2 participantes, até maio de 2001, com um mínimo de sete consultas consecutivas. , $, 5,6,7^{-}$

O modelo proposto consistiu na captação de pacientes que procuraram o CMS para atendimento em qualquer especialidade, e uma triagem com avaliação de fatores de risco para hipertensão arterial e diabetes mellitus foi realizada em todo aquele que assim o permitiu. Os indivíduos que apresentaram medida de pressão arterial acima dos limites preconizados pelo Programa Nacional de Controle da Hipertensão Arterial (PNCHA), em duas verificações, foram inscritos no programa de hipertensão arterial do $\mathrm{CMS}^{4-5}$. Aqueles com dosagem de glicemia venosa acima dos valores de normalidade recomendados pelo Plano de Prevenção e Controle do Diabetes Mellitus no Município do Rio de Janeiro foram, por sua vez, inscritos no programa de diabetes do CMS. De forma alternada, os pacientes foram encaminhados para atendimento no presente projeto ou no ambulatório de clínica médica do CMS, não sendo permitida a migração deles.

- Critérios para a inclusão no Programa de Hipertensão Arterial: adulto, morador, trabalhador ou estudante da área programática do CMS, apresentando níveis tensionais sistólicos $\geq 140 \mathrm{~mm} \mathrm{Hg}$ e/ou diastólicos $\geq 90 \mathrm{~mm} \mathrm{Hg}$ em duas aferições em datas diferentes ou diagnóstico prévio de HAS em uso de medicação anti-hipertensiva.

- Critérios para a inclusão no Programa de Diabetes Mellitus: adulto, morador, trabalhador ou estudante da área programática do CMS, apresentando glicemia venosa de jejum $\geq 126 \mathrm{mg} / \mathrm{dl} \mathrm{em}$ duas ocasiões ou glicemia capilar casual $\geq 200$ $\mathrm{mg} / \mathrm{dl}$ e glicemia venosa de jejum $\geq 126 \mathrm{mg} / \mathrm{dl}$.

- Critério de exclusão: pacientes em acom- 
panhamento no programa do CMS em questão e aqueles que não assinaram o termo de consentimento para participação no projeto.

O atendimento foi realizado por médicos, nutricionistas, enfermeiros e profissionais de educação física, segundo o protocolo do projeto. A primeira consulta foi agendada com o médico, que realizou o diagnóstico clínico e definiu a conduta terapêutica, além de incentivar o paciente a se inscrever na atividade física que apresentava a linguagem do movimento sob a forma de dança. O médico marcava a segunda consulta para o nutricionista, o qual realizava um tipo de atendimento integral, que consistia, além da realização do questionário de consumo alimentar, registro dos dados antropométricos e prescrição dietética, na verificação dos níveis de pressão arterial, glicemia capilar, aderência à terapêutica em geral, resultados de exames e prescrição dos medicamentos, de acordo com a orientação médica em um sistema de interconsulta. O nutricionista agendava a consulta seguinte para o enfermeiro, que desenvolvia também um atendimento integrado, além das ações educativas para controle da doença, encaminhando novamente o paciente para o atendimento médico e, assim, sucessivamente.

Para a avaliação do resultado da atenção dietética, foram consideradas as seguintes variáveis: sexo; diagnóstico clínico; atividade física; faixa etária; escolaridade; peso na admissão no projeto; estatura; cálculo do Índice de Massa Corporal (IMC); pesos relativos ao período entre o primeiro e o sexto mês de acompanhamento; primeira medida de cintura; número de refeições diárias; aplicação do questionário de freqüência em relação ao consumo de frutas e vegetais; exames laboratoriais na admissão ao projeto; níveis tensionais (em hipertensos) e de glicemias de jejum (em diabéticos) nas consultas 1, 2 e 7.

Para a avaliação da aderência ao tratamento, foi utilizado um questionário adaptado e reduzido para seis questões, o qual se baseou no instrumento Testing of the Diabetes Activies Questionnaire (TDAQ) e foi aplicado por entrevistador treinado, não envolvido com prestação dos serviços de saúde. Foram considerados aderentes aqueles que responderam sim, sempre e muitas vezes, às perguntas formuladas no questionário ${ }^{8-9}$.

Para a avaliação da qualidade de vida, foi empregado o questionário Medial Outcome Study 36 - items short-form health survey (SF-36), sendo utilizados os escores de estado geral de saúde, capacidade funcional, aspecto físico, vitalidade, aspecto social, aspecto emocional, saúde mental e dor, para fins de comparação com a resposta ponderal $^{10}$.

Foi realizada uma análise univariada descritiva para avaliar a evolução do peso, das pressões arteriais e das glicemias de jejum. Para comparações entre dois subgrupos, foram utilizados os testes de Mann-Whitney e, para confrontos de médias de dados evolutivos dos pacientes, foi empregado o teste de Dunn. O nível de significância adotado foi de $5 \%$.

A análise multivariada foi feita comparando-se os seguintes dados: peso na primeira consulta $\left(P_{i}\right)$; peso na última consulta $\left(P_{f}\right)$; peso relativo $\left(P_{r}\right)$, obtido na última consulta pela fórmula a seguir;

$$
P_{r}=\frac{\left(P_{f} X\right)}{P_{J}} 100
$$


diferença entre peso inicial e final; (DP); pressões arteriais pré e pós-tratamento e idade - considerando-se como variáveis dependentes cada um dos escores obtidos pelo questionário de qualidade de vida.

\section{III - Resultados}

Foram estudados 116 pacientes, 65 mulheres $(56,1 \%)$ e 51 homens $(43,9 \%)$, dentre os quais $33,7 \%$ eram hipertensos, $25 \%$ tinham hipertensão associada a outras enfermidades, $14,6 \%$ eram hipertensos e diabéticos, $8,6 \%$ eram diabéticos e 18,1\% eram hipertensos, diabéticos, além de sofrerem de outras enfermidades associadas. Oitenta e um por cento deles não realizavam atividade física. Os pacientes analisados tinham de 20 a 65 anos, predominando as faixas de 45 a 54 e de 55 a 64 anos, 31\% cada uma destas. Em relação à escolaridade, $69 \%$ dos pacientes freqüentaram apenas o $1^{\circ} \mathrm{grau}$. $\mathrm{IMC}$ médio de admissão foi de $29,34 \pm 5,22 \mathrm{~kg} / \mathrm{m}^{2}$, sendo que, na faixa de 45 a 54 anos, o IMC médio foi de $30,71 \pm 5,5 \mathrm{~kg} / \mathrm{m}^{2}$. A cintura abdominal foi de $100,30 \pm 8,9 \mathrm{~cm}$ em homens (mediana 99,6 variando de 86 a $120 \mathrm{~cm}$ ) e de 99,2 $\pm 17,0$ (mediana de 97,8 variando de 73 a $130 \mathrm{~cm}$ ). Já o inquérito alimentar mostrou que $52,5 \%$ dos pacientes consomem frutas e $45,6 \%$ vegetais, diariamente.

Como visualizado na figura 1 , houve queda significativa do peso dos pacientes (de 74,4 $\pm 14,1$ para 73,5 $\pm 13,6 \mathrm{~kg} \mathrm{p}<0,0001)$ e do IMC $(29,3 \pm$ 5,2 para $28,84 \pm 5,2$, sendo $p=0,0003$ ) em toda a amostra. Nos obesos, a variação de peso foi de $84,7 \pm 12$ para $83 \pm 12,9$ sendo $p<0,0001$.
Figura 1: Evolução ponderal dos pacientes durante os seis primeiros meses (consultas: de 2 a 7 ).

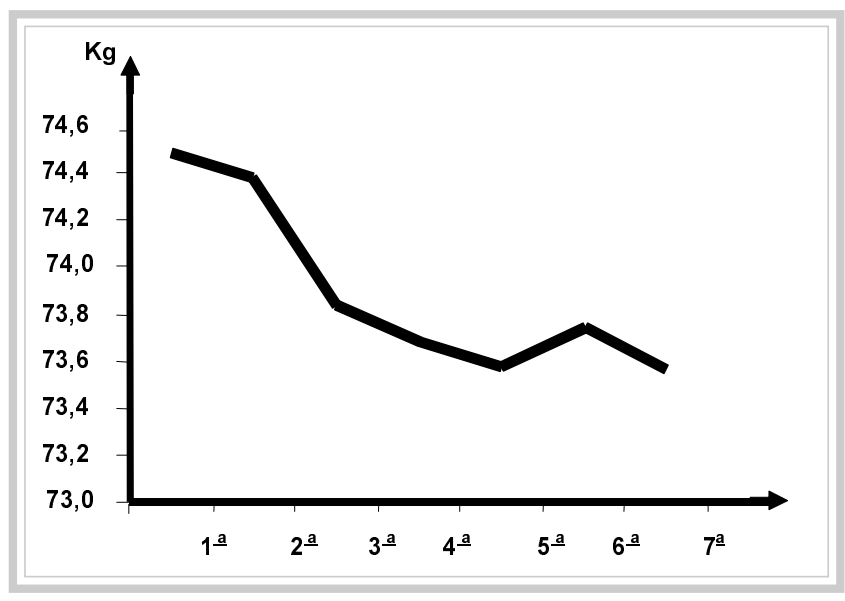

A intervenção nutricional ocorreu na consulta 2. Houve queda significativa do peso $-p<$ 0,0001 . Considerando-se as medianas dos pesos obtidos na consulta 1 (pré-tratamento), houve queda significativa, quando comparadas às consultas de 3 a 7 . Nas comparações entre a consulta 2 (prescrição dietética) e as demais, houve queda relevante a partir da consulta de 4 a 7 .

Na página a seguir, a tabela 1 descreve a curva de pressão arterial dos pacientes hipertensos, antes e depois do tratamento, tendo sido demonstrada redução significante $(p<0,0001)$.

$\mathrm{Na}$ subamostra de pacientes obesos, correspondente a 48 indivíduos $(45,28 \%$ da amostra total), $33(68,75 \%$ dos obesos) responderam positivamente ao controle da pressão arterial (apresentaram valores normais), como pode ser visto na figura 2 .

Curvas ponderais obtidas nos pacientes obesos que responderam ao tratamento da hipertensão arterial normalizando os níveis tensionais ( $n=33$ ), comparadas àqueles que não o fizeram $(n=15)$ - Teste de Dunn. 
Figura 2:

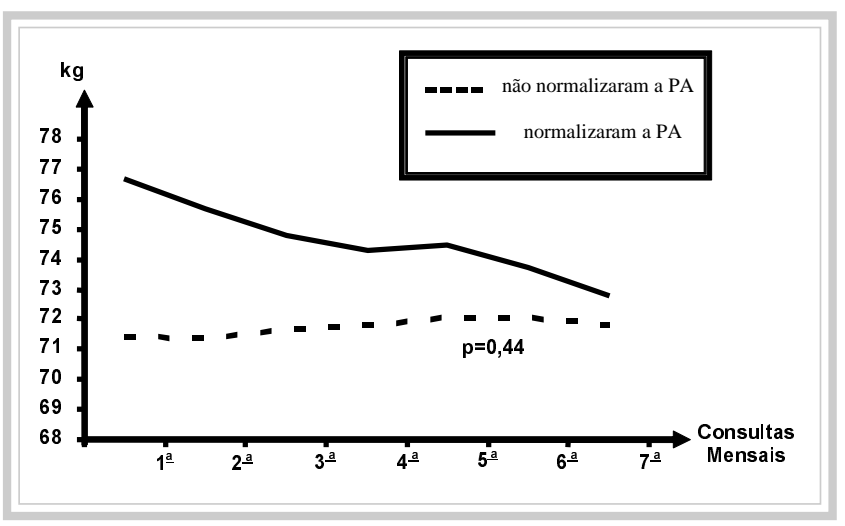

realizados com os pesos aferidos na $1 \underline{a}$ e na $7 \underline{a}$ consulta. O valor de "p" foi > 0,05 para todas as questões, não sendo portanto significativo (Teste de Mann Whitney).

O questionário de qualidade de vida também foi aplicado em 36 pacientes, aleatoriamente. A tabela 3 ilustra a média $( \pm \mathrm{DP})$ dos escores encontrados, e a análise multivariada não demonstrou associações importantes entre os escores de qualidade de vida e os indicadores de

Tabela I - Evolução dos níveis pressóricos dos pacientes acompanhados no Projeto de Hipertensão do CMS

\begin{tabular}{|c|c|c|c|c|c|}
\hline $\begin{array}{c}\text { Pressões } \\
\text { Arteriais }\end{array}$ & $\begin{array}{l}\text { Variáveis } \\
\text { estatísticas }\end{array}$ & $1^{\mathrm{a}}$ consulta & $2^{\mathrm{a}}$ consulta & $7^{a}$ consulta & $\begin{array}{l}\text { Valor de } p^{(*)} \\
\quad\left(1^{a} a 7^{a}\right)\end{array}$ \\
\hline
\end{tabular}

\begin{tabular}{|c|c|c|c|c|c|c|}
\hline $\begin{array}{l}\text { Sistólicas } \\
(\mathrm{mm} \mathrm{Hg})\end{array}$ & $\begin{array}{c}\text { média } \pm \mathrm{DP} \\
\text { mediana (amplitude) }\end{array}$ & $\begin{array}{r}157,2 \pm 23,0 \\
160(110-120)\end{array}$ & $\begin{array}{l}144,6 \pm 22,9 \\
146(70-200)\end{array}$ & $\begin{array}{l}141,8 \pm 22,1 \\
140(70-220)\end{array}$ & $<0,0001$ & $\begin{array}{l}-15,34 \pm 26,46 \\
-15(-90 \text { a } 46)\end{array}$ \\
\hline $\begin{array}{l}\text { Diastólicas } \\
(\mathrm{mm} \mathrm{Hg})\end{array}$ & $\begin{array}{c}\text { média } \pm \mathrm{DP} \\
\text { mediana (amplitude) }\end{array}$ & $\begin{array}{l}95,7 \pm 13,3 \\
96(70-130)\end{array}$ & $\begin{array}{l}90,3 \pm 11,8 \\
90(60-120)\end{array}$ & $\begin{array}{l}87,3 \pm 11,4 \\
86(60-120)\end{array}$ & $<0,0001$ & $\begin{array}{l}-8,40 \pm 14,2 \\
-10(-46 \text { a } 26)\end{array}$ \\
\hline
\end{tabular}

O valor de "p" refere-se a comparações das pressões arteriais seqüenciais. $\triangle \mathrm{PA}=$ Variação da pressão arterial no período estudado. DP = desvio padrão. Os valores de " $p$ " foram significativos $(p<0,0001)$ tanto para pressão sistólica quanto para diastólica, com significância de 0,05 (consulta $1 \times 7$ ) e 0,0001 (consulta $2 \times 7$ ) para ambas. (*) Teste de Dunn.

O questionário de aderência ao tratamento foi aplicado em 39 pacientes escolhidos aleatoriamente. A tabela 2 ilustra a perda de peso relativo $\left(P_{r}\right)$ dos pacientes que aderiram ao tratamento e dos que não aderiram, avaliados por questões dirigidas.

Foram considerados aderentes aqueles pacientes que responderam sempre ou muitas vezes e não-aderentes aqueles que responderam às vezes, raramente ou nunca. Os cálculos foram resposta ponderal.

A análise da resposta de controle dos diabéticos foi prejudicada pelo reduzido número de pacientes (17), nos quais houve uma queda da glicemia capilar casual de 247,2 $\pm 101,8$ mg\% (mediana 207, variando de 139 a 483 mg\%) para $198,1 \pm 79,8 \mathrm{mg} \%$ (mediana de $174 \mathrm{mg} \%$, variando de 130 a $478 \mathrm{mg} \%$ ) entre a primeira e a última consulta, porém não significativa $(p<0,07)$. Houve também redução no peso médio dos pacientes diabéticos de 
Tabela 2: Percentual de perda de peso relativo nos pacientes acompanhados no Projeto de Hipertensão do CMS, segundo a adesão ao tratamento.

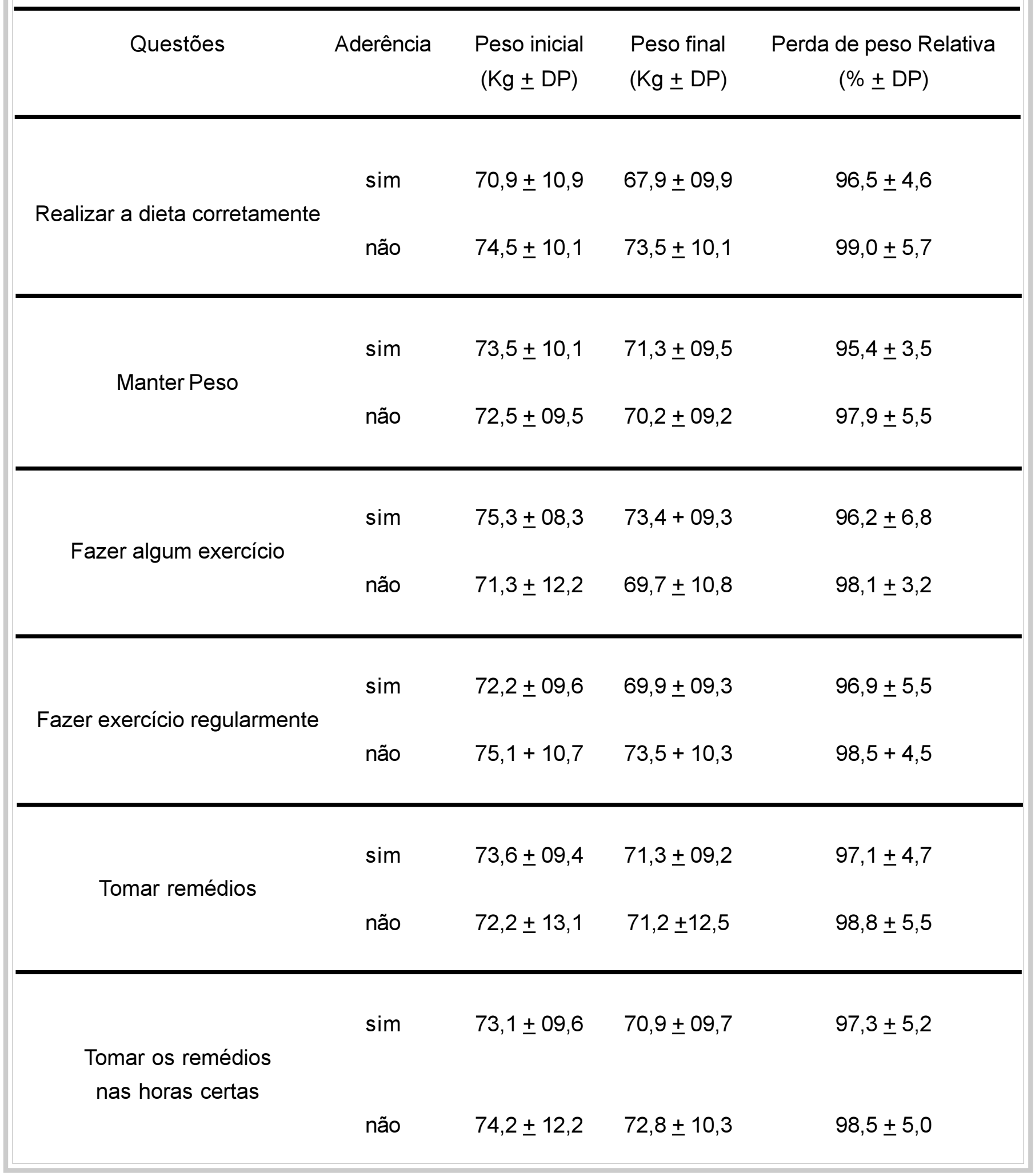


Tabela 3: Escores obtidos no questionário de qualidade de vida aplicado a 36 pacientes acompanhados no Projeto de Hipertensão do CMS.

\begin{tabular}{lcc}
\hline Variável & Média + DP & Mediana (Amplitude) \\
\hline Estado geral de saúde & $66,16 \pm 23,02$ & $69,5(05-100)$ \\
Capacidade funcional & $74,86 \pm 23,55$ & $80,00(05-100)$ \\
Aspecto Físico & $63,19 \pm 39,40$ & $75,00(00-100)$ \\
Vitalidade & $41,88 \pm 12,61$ & $40,00(12-083)$ \\
Aspecto Social & $52,22 \pm 21,39$ & $50,00(10-080)$ \\
Aspecto Emocional & $68,05 \pm 37,75$ & $83,0(00-100)$ \\
Saúde Mental & $60,69 \pm 16,26$ & $62,5(20-095)$ \\
Dor & $58,65 \pm 23,68$ & $50,5(22-100)$ \\
\hline
\end{tabular}

Os escores para todas as variáveis podem ser de zero a cem. Quanto maior o escore obtido para a variável, melhor o desempenho, exceto para dor.

$74,5 \pm 16,4$ (mediana 71,4, variando de 52,0 a 107,7), na consulta 1 , para $72,6 \pm 14,7$ (mediana 71,0, variando de 56,2 a 106,6), na consulta 7, mas essa diferença não foi significativa $(p=0,88)$.

\section{IV - Discussão}

A relevância do estudo se relaciona ao fato de que houve uma mudança no tipo de inserção profissional do nutricionista no modelo de atendimento à saúde proposto, ampliando muito seu papel, cujo objeto de trabalho, habitualmente, está restrito aos cuidados dietéticos. Neste modelo, sua função é ampliada, havendo uma intervenção mais global no tratamento da hipertensão arterial e do diabetes mellitus. Desta forma, o controle da pressão arterial, da glicemia e dos níveis lipêmicos, a observação da aderência ao tratamento de modo geral, a detecção dos efeitos colaterais tanto da dieta quanto das drogas utilizadas e a prescrição da terapia são ações que permitem a abordagem integral de todos os profissionais envolvidos no tratamento dessas duas doenças, tão freqüentes na população, e as quais esperamos que exerçam um maior impacto nos resultados esperados.

Neste estudo, não houve preocupação de se avaliar os resultados desta ação comparandoa com o modelo tradicional de atendimento focalizado no médico, parte de um projeto maior, 
cujos resultados estão sendo analisados e serão oportunamente publicados, cabendo, aqui, apenas a análise de nossa prática nesta nova inserção profissional, em um estudo piloto com 116 pacientes.

As características gerais de nossa amostra são semelhantes às estudadas pela maioria dos autores, havendo a mesma prevalência de hipertensão arterial em ambos os sexos, aumentando com a idade, principalmente após 45 anos, em pacientes que em sua imensa maioria são sedentários e com diferentes graus de excesso de peso ${ }^{12}$.

O consumo alimentar no Brasil parece estar modificando-se, havendo redução progressiva da ingestão de vegetais e frutas. Cerca de metade de nossos pacientes apresentaram frutas e legumes em seu cardápio, fato que merece uma melhor investigação, já que há evidências de grandes modificações de atitudes e valores em relação à cultura alimentar urbana, a qual tem revelado que o consumo desses alimentos tem perdido força e sido substituído por uma dieta carente deles. ${ }^{13}$

No presente estudo, houve queda significativa tanto do peso quanto do IMC, considerando-se toda a amostra, e particularmente nos indivíduos com sobrepeso e obesidade, que constituíram $87 \%$ dos casos. O pique máximo de perda de peso ocorreu no terceiro mês após a intervenção nutricional, tendendo à manutenção após este tempo.

Houve queda significativa dos níveis tensionais, tanto sistólicos quanto diastólicos, conseqüentes do tratamento medicamentoso e restrição de sódio, observado pela diferença significativa de queda de pressão entre as consultas 1 e 2 . O estudo randomizado controlado e multicêntrico DASH ${ }^{14}$ já havia demonstrado o efeito da dieta com redução de quantidades de sal, potencializando os efeitos medicamentosos no tratamento da hipertensão arterial. A prescrição dietética é tão mais importante quanto mais precoce sua realização, particularmente em obesos, nos quais os efeitos da perda de peso são mais tardios. No presente estudo, provavelmente a redução de sódio deve ter contribuído para as quedas significativas de PA observadas ao final do primeiro mês.

A queda dos níveis de pressão arterial foi mais significativa, no entanto, comparando-se as consultas 1 e 7 , quando os efeitos da dieta prescrita em relação à curva ponderal foram mais evidentes (tabela 1). Houve diminuição significativa do peso nos obesos que tiveram a pressão arterial normalizada em relação àqueles que não tiveram.

Chamou a nossa atenção que, nos obesos que apresentaram normalização da pressão, o peso final ainda foi mais alto do que o daqueles que se mantiveram hipertensos (figura 2), ressaltando a importância da perda ponderal relativa individual no controle da hipertensão. Esses fatos demonstram a importância da redução ponderal como coadjuvante no tratamento da hipertensão arterial em obesos, independente do peso inicial ou final. É importante definir, portanto, metas de perda de peso factíveis para obesos, reavaliando-as progressivamente, ao longo do tratamento. Essa conduta pode garantir maior efetividade ao tratamento da hipertensão arterial nestes pacientes. A prescrição dietética para hipertensão leve pode, inclusive, constituir-se como único tratamento, 
como demonstrado por Jorge ${ }^{15}$ em seu estudo com 40 pacientes com este grau de hipertensão, no qual $45 \%$ apresentaram os níveis tensionais reduzidos apenas com dieta. Na nossa amostra, apenas 12 pacientes com hipertensão arterial leve tinham IMC igual ou superior a 25 , o que tornou esta análise inconclusiva.

Kumanvika et al. ${ }^{16}$ avaliaram afro-americanos com índice de massa corporal (IMC) entre 30 e 50, durante dez meses, em uma clínica para redução de peso, e apontaram como fatores preditores independentes de abandono da dieta a idade menos avançada $(p<0,001)$, escolaridade $<12$ anos $(p=0,008)$ e a ocupação sedentária $(p=$ 0,028 ). O maior percentual de falhas no tratamento esteve associado aos níveis mais elevados de pressão diastólica ( $p=0,012)$, ao maior ganho de peso nos últimos seis meses $(p=0,027)$ e a uma tendência na percepção da dieta como algo que exige muito esforço ( $p=0,017)$. Este último fato corrobora a importância do planejamento de metas alcançáveis de redução ponderal em obesos, o que propicia a melhora da percepção do esforço exigido para a aderência à dieta. Em nossa amostra, apesar da escolaridade (69\% da amostra não atingira o $2^{\circ}$ grau) e do sedentarismo (81\% não praticava exercícios físicos), houve resultados de queda de peso significativos no tempo do estudo. Uma nova avaliação após um período maior de observação necessita ser feita.

É importante enfatizar que o estudo considerou como meta para controle da hipertensão arterial níveis de $140 \times 90 \mathrm{~mm} \mathrm{Hg}$, e apenas $42,1 \%$ atingiram este objetivo no período estudado. Welch ${ }^{17}$ obteve bom controle da hipertensão arterial em $27 \%$ dos pacientes atendidos somente por médicos em serviços de saúde, após um ano de acompanhamento. Esse fato sugere que as diferentes abordagens da equipe multidisciplinar no novo modelo proposto podem favorecer melhores resultados no tratamento da hipertensão arterial.

O estudo comunitário de Stanford trabaIhou com três grupos: 1) com população controle; 2) com intervenção da mídia em ações educativas e 3) utilizando ações educativas e abordagem individualizada para pacientes com alto risco de desenvolver coronariopatia. Foi demonstrada queda significativa do risco cardiovascular nas duas últimas em relação à primeira; também houve queda considerável na faixa de 6,6 a 8,9 $\mathrm{mm} \mathrm{Hg}$ da pressão sistólica, nos grupos 2 e 3 em relação ao grupo controle e nenhuma diferença signifi- cativa na queda da pressão diastólica entre as três populações. ${ }^{18}$

O Programa de Minnesota, também envolvendo três populações, com abordagem multimídia, obteve apenas um modesto declínio nos níveis tensionais, sem significância estatística, até após seis anos. ${ }^{19}$ Resultados semelhantes foram obtidos pelo "Pawtucket Heart Health Program", demonstrando as dificuldades no controle da doença. ${ }^{20}$

No presente estudo, os pacientes que aderiram ao tratamento (88\%), apresentaram reduções expressivas de peso em relação àqueles que não o fizeram, considerando-se todas as variáveis pesquisadas: dieta, exercícios e tratamento medicamentoso (tabela 2). Esses resultados não foram, entretanto, significativos, provavelmente pelo menor tamanho da amostra de pacientes que responderam ao questionário.

Níveis relativamente ruins de qualidade de 
vida referentes aos aspectos sociais, dor e vitalidade foram observados na população estudada. Tendo em vista que grande parte dos pacientes era de baixa renda, submetida a constante violência, os achados referentes aos aspectos sociais podem refletir esta condição. É relevante o baixíssimo escore obtido no ítem vitalidade. Como este parâmetro refere-se à disposição física (vigor) para realização de atividades em geral, ele pode ter sido muito influenciado pelos aspectos sociais. Em relação à dor, não encontramos justificativa, tendo em vista que a hipertensão arterial e o diabetes leve raramente se associam a essa queixa. Os aspectos emocionais e a saúde mental obtiveram escores moderados.

A capacidade funcional teve o melhor escore médio obtido, seguido do estado geral de saúde e do aspecto físico, variáveis analisadas com base na percepção dos próprios limites corporais. Como tanto a hipertensão quanto o diabetes moderados ou leves não são doenças incapacitantes, nesta fase evolutiva, esses resultados ficaram dentro do esperado.

Não houve influência das respostas ponderais obtidas sobre as variáveis de qualidade de vida, o que, acreditamos, pode ter ocorrido pela variação relativamente pequena do peso no curto período estudado (sete meses).

Em relação aos diabéticos, o pequeno número de pacientes prejudicou a análise dos resultados, embora já se possa perceber tendência de queda da glicemia de jejum e redução do peso dos pacientes. Desta forma, estudos mais detaIhados são necessários para o esclarecimento do papel da intervenção nutricional da equipe mul- tiprofissional no diabetes mellitus no modelo proposto.

\section{V - Conclusões}

A intervenção nutricional para atendimento a hipertensos em uma unidade de atenção primária contribuiu para uma boa resposta ao tratamento dessa doença. A perda ponderal parece ser um fator mais importante do que o peso em si na diminuição da pressão arterial em obesos. Assim, metas de perda de peso factíveis e contínuas devem ser estipuladas ao longo do programa alimentar.

\section{VI - Referências}

1- Baltazar J, Natario A. An evaluation of the quality of medical care for hypertensive patients. $A$ review of the record of explicit criteria and standard in the clinics of District of Lisbon. Acta Med Port 1993; 6 (10): 431-38.

2- Ostenson CG. Pathophysiology of type 2 diabetes mellitus: an overview. Acta Physiol Scand 2001; 171(3): 241-7.

3- Halfoun VLRC, Araujo MC, Malveira, EAP, Gonçalves MC, Griep RH, Mattos D et al. Custo/ efetividade de um modelo assistencial multidisciplinar nos programas de hipertensão arterial e diabetes mellitus em uma unidade de saúde do município do Rio de Janeiro. In: Anais do VI Congresso Brasileiro de Saúde Coletiva, Ciência e Saúde Coletiva, 2000; Salvador, Brasil. Rio de Janeiro: ABRASCO; 5 (supl): 193, 2000.

4- Brasil. Ministério da Saúde. Secretaria Nacional de Programas Especiais de Saúde. Divisão Nacional de Doenças Crônicasdegenerativas. Manual de Diabetes. Brasília (DF): 
Ministério da Saúde; 1990.

5- Brasil. Ministério da Saúde. Secretaria Nacional de Doenças Crônicas-Degenerativas. Programa nacional de Educação e Controle da Hipertensão. Normas técnicas para o Programa Nacional de Hipertensão Arterial (PNECHA). Brasília (DF): Centro de Documentação do Ministério da Saúde; 1998.

6- Brasil. Ministério da Saúde. Secretaria Nacional de Doenças Crônicas-Degenerativas. Plano de Assistência e Reorganização da Hipertensão Arterial e Diabetes Mellitus, Brasília (DF): Centro de Documentação do Ministério da Saúde; 2002.

7- World Health Organization. Definition, diagnosis and classification of diabetes mellitus and its complications. Reports of a WHO consultation. Part 1: diagnosis and classification of diabetes mellitus. Washington, D.C.: WHO; 1999.

8- Halfoun VLRC, Araujo MC, Mattos D, Falcão RC, Barreto MR, Zisman VL et al Avaliação de dois modelos de atendimento nos programas de hipertensão arterial e de diabetes mellitus em uma unidade de saúde do município do Rio de janeiro: a aderência ao tratamento. In: Anais do VII Congresso Brasileiro de Saúde Coletiva, Ciência e Saúde Coletiva, 2003; Brasília DF, Brasil. Rio de Janeiro: ABRASCO; 2003; 8 (1): 236.

9- Hernandez CA. The development and pilot testing of the Diabetes Activities Questionnaire (TDAQ): an instrument to measure adherence to the diabetes regimen. Appl Nurs Res. 1997; 10(4): 202-11.

10- Ware JE, Snow RK, Kosinski M, Gandek B. SF-36 Health Survey. Manual and Interpretation Guide. Boston: Ed. The Health
Institute. New England Med. Center, Man. 1993. Nimrod.

11- Ciconelli RM. Tradução para o português e avaliação do questionário genérico de avaliação de qualidade de vida "medial outcome study 36-item short-form health survey (SF36)" [tese]. São Paulo: Escola Paulista de Medicina Universidade Federal de São Paulo, 1997.

12- Chavez Jr NHC, Negrão CE, Amodeo C, Cunha CP, Lima EG, Monego ET, et al. Hipertensäo arterial: tratamento näomedicamentoso ou modificaçöes do estilo de vida. Rev. Bras. Hipertensão 1999; 6 (1): 81-85.

13- Diez Garcia RW. A comida, a dieta, o gosto: mudança na cultura alimentar urbana [Tese]. Säo Paulo: Instituto de Psicologia - Universidade de São Paulo, 1999.

14- Olmos, RD \& Benseñor, IM. Dietas e hipertensäo arterial: Intersalt e estudo DASH. Rev. Bras. Hipertens 2001; 8(2):221-224.

15- Jorge MIE. Tratamento dietético da hipertensäo arterial leve [dissertação]. São Paulo: Faculdade de Saúde Pública - Departamento de Nutrição - Universidade de São Paulo, 1999.

16- Kumanyika SK, Shults J, Whitt MC, Cunningham JE and Floyd F. Predictors of attendance among African Americans in a primary care weight control program. Am Heart J 2002; 144(5): 15 (abstract).

17- Welch VLL \& Hill MN. Effective strategies for blood pressure control. Cardiology Clinics 2002; 20 (2).

18- Williams PT, Fortmann SP, Farqhuar JW, Varady A and Mellen S. A comparison of statistical methods for evaluating risk factor change in community-based studies: an example from the 
Stanford Three-Community Study. J Chronic Dis 1981; 34:565-71.

19- Luepker RV, Murray DM, Jacobs Jr DR, Mittelmark MB, Bracht N, Carlaw R et al. Community education for cardiovascular disease prevention: risk factor changes in the Minnesota Heart Health Program. Am J Public Health 1994; 84:1383-93.

20- Winkleby MA, Feldman HA and murray DM. Joint analysis of three U.S. community intervention trials for reduction of cardiovascular disease risk. J Clin Epidemiol 1997;50:645-58.

Este trabalho contou com o apoio financeiro da Fundação Universitária José Bonifácio/UFRJ.

Endereço de Correspondência:

Odaleia Barbosa de Aguiar

PAPS - FM-UFRJ

Rua Laura de Araújo, 30

Cidade Nova - Rio de Janeiro

CEP 20211-170, RJ

Endereço eletrônico:

papsfm@ig.com.br 